\title{
TEACHING INTERFACING FOR REAL-TIME SYSTEMS: NOW AND TOMORROW
}

\author{
Witold Kinsner, Dario Schor, and Ken Biegun \\ Department of Electrical and Computer Engineering \\ University of Manitoba, Winnipeg, MB, Canada R3T 5V6 \\ \{kinsner@ece.umanitoba.ca | dario_schor@umanitoba.ca\}
}

\begin{abstract}
Teaching undergraduate students about interfacing of microprocessors and microcontrollers in real-time systems is challenging because the circuits have moved from medium to increasingly higher frequencies (multimega- and giga hertz), while wired interfacing has been augmented with wireless interfacing. This paper describes an attempt to accommodate the changes in an undergraduate course called Microprocessor Interfacing ( $\mu \mathrm{I})$ that has been offered at the University of Manitoba for many years now [1-4].

The course presents real-time wired and wireless interfacing of microcontrollers, microprocessors, and microcomputers to the external world, including interfacing of input/output (I/O) devices with minimum hardware and software, as well as data acquisition with and without microprocessors, data communications, transmission and logging with embedded computers. The following topics are covered: (i) introduction on computing, architectures, processors, and technologies, (ii) architecture and organization of small computer buses, and synchronization of data transfers on local buses (iii) digital input and output (I/O), (iv) digital-to-analog (D/A) and analog-to-digital (A/D) signal conversions and converters, ( $\mathrm{v}$ ) and interfacing aspects in data communications, including encoding, modulation, error detection and forward error protection. The course also includes (a) demonstrations of bus architectures, modules, systems, and new devices, as well as (b) updates on new concepts, technologies, protocols, and software. The laboratories are innovative in terms of three levels of complexity: the Tier1 level includes five 3-hour standard labs designed for all students, the Tier2 is designed for more experienced students, and the Tier3 level lab is designed as a project for groups of students with demonstrated prior design and implementation experience [3].

Tomorrow's teaching will require a deeper exposure to high-speed circuits, as the impact of the distributed nature of signals in circuits increases due to the circuit interconnections acting as transmission lines. This requires software-based simulation and emulation of designs. Embedded systems on a chip may mitigate the problem to a degree. Course laboratories may also change because of the inexpensive equipment such as logic analyzers, protocol analyzers [5], and computer-based digital oscilloscopes. Since the test cost of such equipment is within a range of a student, laboratories-athome may also become a reality.

The paper provides a description of a class structure of an interfacing course today, and identifies changes required by future material in such as course. An example of an implementation of a change is also provided.
\end{abstract}

\section{References}

[1] W. Kinsner, Microcontroller, Microprocessor, and Microcomputer Interfacing for Real-Time Systems. Lecture Notes. Winnipeg. MB: University of Manitoba, 2011, 643 pp.

[2] W. Kinsner, Laboratories for Microcontroller, Microprocessor, and Microcomputer Interfacing for Real-Time Systems. Lab Notes; 2011, 92 pp.

[3] Witold Kinsner, and Kalen Brunham, "A multi-tier laboratory scheme in the interfacing for real-time systems course," in Proc. 14th Can. Conf. Computer Engineering Education, CCCEE01 (Fredericton, NB; May 30-June 1, 2001) 64 pp., 2001.

[4] Jonathan W. Valvano, Embedded Microcomputer Systems: Real Time Interfacing. Stamford, CT: CENGAGE Learning, 2012 (3rd ed.), 793 pp. \& CD-ROM. This book covers design methodologies with examples, using the Motorola 9S12 machine, with many practical examples. The CD-ROM includes a simulator to show internal and I/O activities, and many links to data sheets. \{ISBN 978-1-111-42625-5\}

[5] Witold Kinsner, Dario Schor, and Kathryn, "Reinforcing the design foundation of asynchronous serial data communications using logic and protocols analyzers," in Proc. of the Canadian Engineering Education Conference (St. John's, NL; June 6-8, 2011) 8 pp., 2011.

Conference Topics: (1) Classroom innovation; (2) Undergraduate education.

Keywords: Computer engineering course; laboratory innovation; teaching high-speed circuits.

CEEA Conf. 2012; Paper 105

University of Manitoba, Winnipeg, MB
CEEA12_Abstr_Interf-v20.doc

-1 of $1-$
February 16, 2012 